
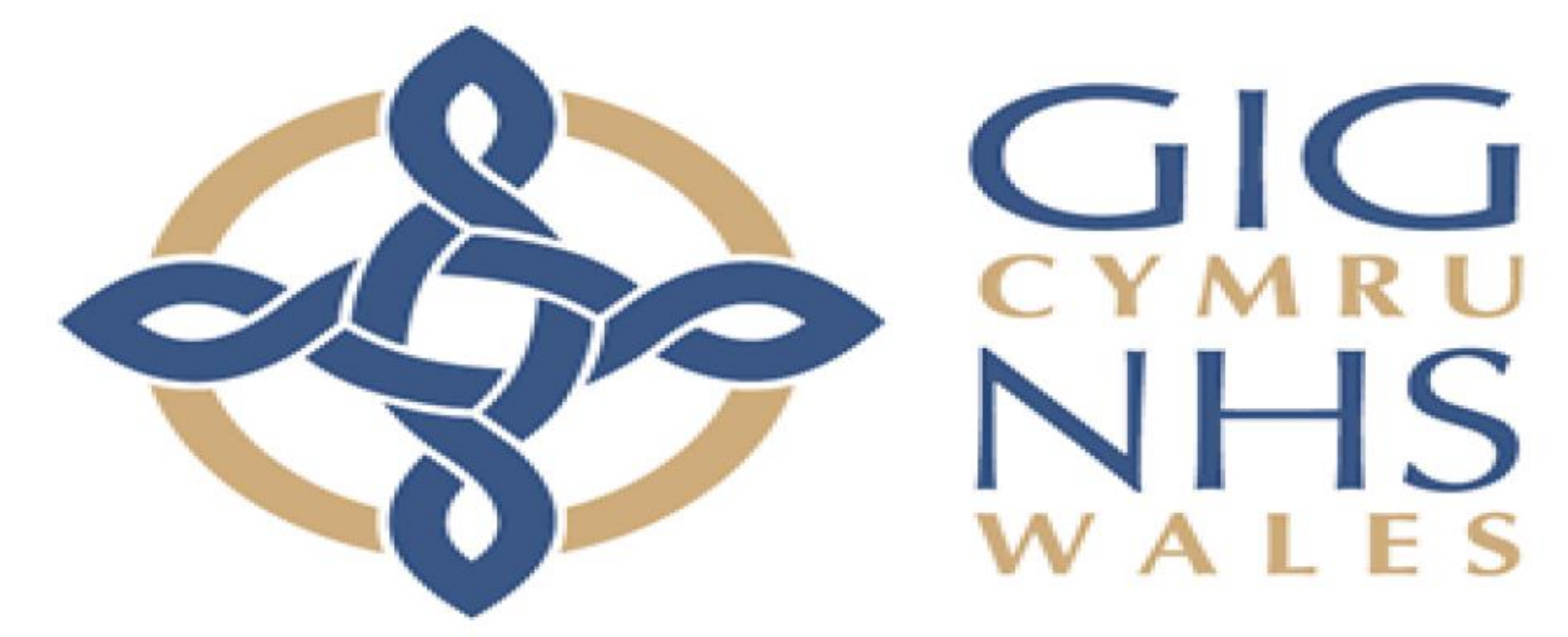

\author{
Bwrdd lechyd Prifysgol \\ Betsi Cadwaladr \\ University Health Board
}

\title{
Drug-induced hepatitis in a patient with Graves” disease
}

Seifeldin Yahia, Nicola Sommers, Betsi Cadwaladr University Health Board,Ysbyty Gwynedd, Bangor

\section{Contents}

A 52 year old lady presented with a history of weight loss, tachycardia and tremor. She was diagnosed with primary hyperthyroidism; TFTs were TSH $<0.02 \mathrm{mu} / \mathrm{l}$ and FT4 $>100 \mathrm{pmol} / \mathrm{I}$, LFTs were mildly deranged (ALT 79 $\mathrm{u} / \mathrm{l}, \mathrm{ALP} 101 \mathrm{u} / \mathrm{l})$. TSH receptor antibodies were positive, suggestive of Graves' disease.

The patient was started on carbimazole, initially at $10 \mathrm{mg}$ TDS which was titrated down to $5 \mathrm{mg}$ TDS. Repeat LFT showed ALP at $219 \mathrm{u} / \mathrm{l}$ and a fluctuating ALT between $58 \mathrm{u} / \mathrm{l}$ and $141 \mathrm{u} / \mathrm{l}$ over the first 5 months on carbimazole.

Repeat LFT during a routine clinic appointment showed grossly deranged LFTs, with ALT of $1001 \mathrm{u} / \mathrm{l}$ and ALP of $306 \mathrm{u} / \mathrm{l}$. Carbimazole was stopped immediately. Full hepatic screen was unrewarding a part from positive smooth muscle autoantibodies. This could suggest a diagnosis of autoimmune hepatitis, which is associated with Graves' disease, but given that ALT was $>30 x$ normal, whilst ALP was only $2 x$ normal, this picture is more in keeping with acute hepatitis secondary to carbimazole.

Only one week after stopping carbimazole the patient rapidly became thyrotoxic, with FT4 of $60.7 \mathrm{pmol} / \mathrm{l}$ and FT3 of $30.5 \mathrm{pmol} / \mathrm{l}$. She was started on lithium and lugol's iodine in order to normalize TFTs prior to thyroidectomy. LFTs normalized over a period of 4 weeks after stopping carbimazole.

The patient underwent total thyroidectomy; histology showed diffuse hyperplasia. Post-operatively she had right vocal cord paralysis but this has resolved spontaneously. The patient is now on replacement thyroxine.
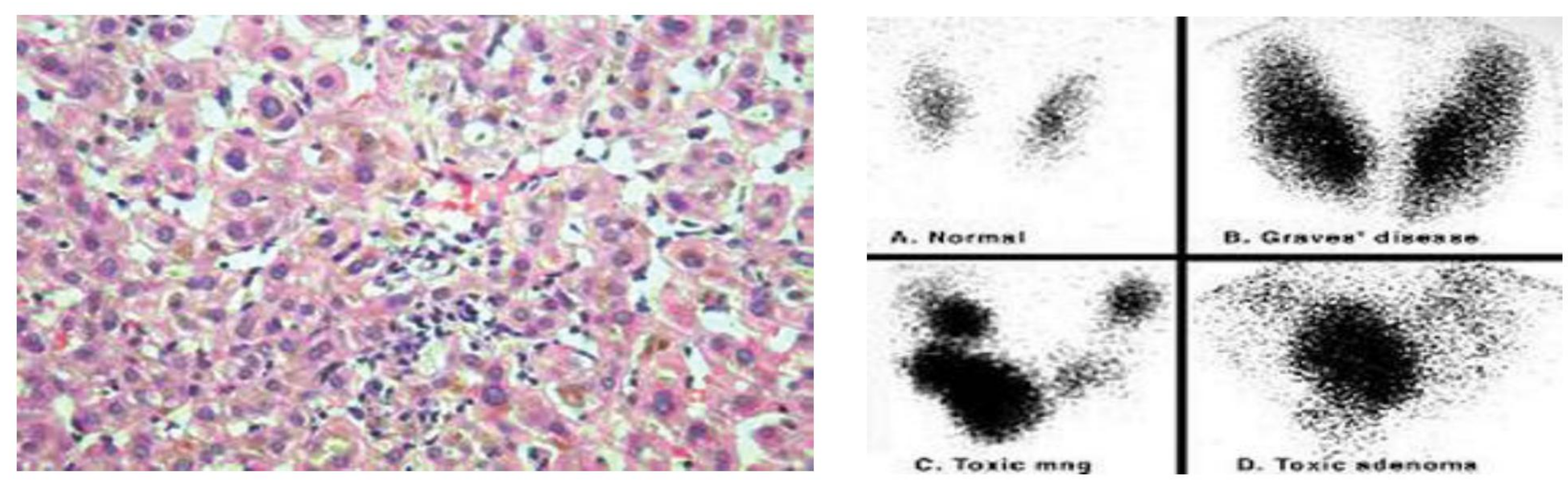

\section{Conclusion:}

Hepatotoxicity is rare but serious side-effect of carbimazole. It is important to consider all causes of hepatitis. Where there is concern about the cause of hepatitis, surgery may be preferable when compared to changing to another antithyroid drug. 